\title{
Cytogenetics of Rutaceae. V. High chromosomal variability in Citrus species revealed by CMA/DAPI staining
}

\author{
M. GUERRA \\ Departamento de Genética, Centro de Ciências Biológicas, Universidade Federal de Pernambuco, Recife 50.739, PE, \\ Brazil
}

\begin{abstract}
The CMA/DAPI chromosome banding pattern of six Citrus species was analysed. All of them showed a large amount of heterochromatin and heterozygosity. Most of the heterochromatin was $\mathrm{CMA}+/ \mathrm{DAPI}-$ and was located mainly in the terminal region of the long arms. Each individual was heterozygous for at least one chromosome pair and each species presented a different banding pattern. The greatest amount of information on karyotype variation can be obtained from the four largest pairs. Pairs I and III showed the highest amount of heterochromatin and heterozygosity whereas pair II was highly stable and almost completely lacked CMA+ heterochromatin. On the other hand, some features common to all species suggest a strong chromosome homoeology conservation. The CMA staining revealed some chromosome markers for each Citrus species that may be very useful in the identification of zygotic versus nucellar embryos, as well as for further evolutionary studies.
\end{abstract}

Keywords: chromosome markers, Citrus, CMA banding, CMA/DAPI staining, heterochromatin, karyotypes.

\section{Introduction}

The genus Citrus is known for its great genetic variability and almost unsolvable infrageneric systematics (Barrett \& Rhodes, 1976). The origins of both problems seem to lie in the high frequency of nucellar embryony (agamospermy) and the facility of interspecific and intergeneric hybridization with total or partial fertility. Hybrids between closely related species are often highly vigorous and nucellar embryony can preserve any heterozygosity and so it is generally assumed that a combination of these two factors has created many clonally propagated entities with large genomic diversity. On the other hand, in commercial varieties, zygotic seedlings present many undesirable genotypic combinations with some degree of inbreeding depression and nucellar embryony in these becomes one of the most important hindrances to Citrus breeding (reviewed by Cameron \& Frost, 1968).

The genetic variability commonly observed is related to phenotypic characteristics such as fruit size and shape, tree vigour, susceptibility to diseases, level of acidity in fruits, etc. Isoenzyme analysis has found similar genetic variability at the biochemical level even in morphologically uniform seedling populations (Moore \& Castle, 1988). Such an approach has also been useful to distinguish zygotic from nucellar seedlings (Iglesias et al., 1974; Ashari et al., 1988). As we are concerned with the cytogenetic analysis of the genus, we are interested in identifying its chromosomal variability and in looking for a further approach to the genetic characterization of species or clones of Citrus.

Citrus chromosomes seem initially to be unsuitable for detailed analysis. The chromosome number $2 n=18$ is constant in the genus, with the exception of some cultivated polyploids (Krug, 1943; Cameron \& Frost, 1968). Furthermore, the mitotic chromosomes are very small $(1-4.0 \mu \mathrm{m})$ and most of them are similar in morphology (Frost, 1925; Krug, 1943; Sharma \& Bal, 1957).

Most of the recent reports on Citrus cytogenetics have been confined to descriptions of meiotic behaviour (Agarwal, 1987a, b; Sellito-Boaventura \& Pio, 1989) or mitotic chromosome number in plants manipulated in vitro (Grosser et al., 1988; Kobayashi et al., 1991). Some authors have also analysed Citrus chromosomes with C-banding (Guerra, 1985; Liang, 1988; Wei et al., 1988). Most of the chromosomes had 
many heterochromatic blocks essential for karyotype identification but this method was not satisfactory as the chromosome morphology was markedly affected and the banding pattern showed some variation, even on a single slide, because of artefacts.

In this paper we report the chromosomal banding patterns of some Citrus species stained with the fluorochromes Chromomycin A (CMA) and 4'-6-diamidino-2-phenylindole (DAPI) which permit identification of several chromosome markers for most species or clones.

\section{Materials and methods}

The plants investigated were grown in pots in the Botanical Garden of the University of Vienna, except Citrus limonia Osbeck which was obtained from a local market in Vienna. Root tips from young plants of $C$. aurantium L., C. grandis Osbeck, C. limon (L.) Burm.f. and $C$. sinensis Osbeck and an adult fruiting individual of $C$. medica $\mathrm{L}$. were collected from the pots. Seeds of C. limonia were germinated in Petri dishes and root tips from several seeds were collected for cytological analysis. All root tips were pretreated with 8-hydroxyquinoline $(0.002 \mathrm{M})$ at $12^{\circ} \mathrm{C}$ for $5-6 \mathrm{~h}$, fixed in ethanolacetic acid $(\mathrm{v} / \mathrm{v}, 3: 1)$ and stored at $-20^{\circ} \mathrm{C}$ until required.

For slide preparation, the root tips were washed twice in distilled water (10 min each), digested with a 2 per cent cellulase-pectinase solution $(30 \mathrm{~min})$ and squashed in 45 per cent acetic acid. After coverslip removal the slides were aged for 3 days, stained with CMA for $1 \mathrm{~h}$, counterstained with DAPI for $30 \mathrm{~min}$ and mounted in McIlvaine's $(p \mathrm{H}$ 7.0) buffer-glycerol v/v 1:1 (Schweizer, 1976; Deumling \& Greilhuber, 1982). Photomicrographs were taken on Kodak Tri-X Pan film. Drawings from karyotype analysis were made using amplified projection from film negatives on the best metaphases of each species.

\section{Results}

The Citrus species analysed showed a large number of telomeric, and rarely proximal, bands with enhanced CMA and quenched DAPI fluorescence (CMA+/ DAPI -). The telomeric bands were very variable in size and were preferentially located on the long arms. The interphase nuclei displayed a proportionally large number of CMA+ chromocentres (Fig. $3 \mathrm{c}$ and e). They seemed to correspond to the largest chromocentres observed in conventionally stained nuclei (see Guerra, 1985, 1987). Many small CMA-/DAPI+ chromocentres were also present in interphase nuclei although DAPI + bands were not observed on prophase or metaphase chromosomes (Fig. 3d and f).

All chromosome complements showed a distinct banding pattern that was consistent between cells of each individual. Notably, each karyotype was heterozygous for at least one chromosome pair. The CMA+ banding pattern of each chromosome was classified into one of the following types to simplify karyotype description and comparison (Fig. 1):

A: two telomeric and one proximal band.

$\mathrm{B}$ : one telomeric and one proximal band.

C: two telomeric bands.

$\mathrm{D}$ : one large telomeric band.

E: one small telomeric band.

F: bands absent or only very fine and not always visible.

To establish chromosome morphology and size, the CMA and DAPI pictures of each measured chromosome complement were used. The CMA and DAPI images of the same cell were not easily superimposed. The CMA-stained picture gives an enlarged size of the CMA+ blocks whereas the DAPI one makes the euchromatin seem broader and larger than it really is. The chromosome pairs were ordered in decreasing size from the largest (pair I) to the smallest (pair IX). The centromeric position was better observed with DAPI staining but in some cases could not be precisely determined.

The karyotype of C. aurantium was homozygous for all chromosomes except pair III (CD). The D chromosome sometimes contained an additional fine centromeric CMA+ band and was smaller than its $\mathrm{C}$ homologue. Pair I was a CC type characterized by a proximal region faintly stained with CMA, deeply DAPI negative and sometimes stretched (Fig. $2 \mathrm{a}$ and b). Pair II (FF) is noteworthy because it was the only large chromosome almost equally stained except for a very fine terminal band not always present. This band was present in the same way in pair II of all other
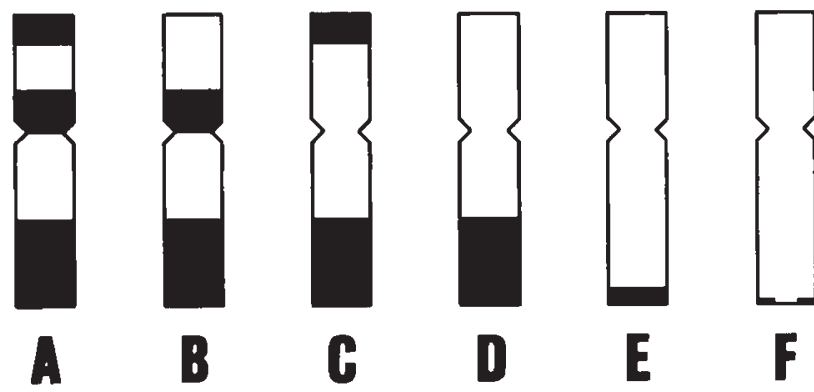

Fig. 1 Main chromosome types in Citrus according to the size and position of CMA+ bands. 
species. Pairs IV and V were DD, VI, VIII and IX were FF and VII was EE.

The karyotype of Citrus grandis was characterized by three main features: (i) an AA pair I, with a large terminal band in the long arm and a smaller telomeric one in the short arm; (ii) an AC pair III, also heteromorphic for the chromosome size; and (iii) a CC pair IV (Fig. 2c and d). A fine proximal band was often observed in the $\mathrm{C}$ type chromosomes of pair IV and sometimes also in the III C. Chromosome pairs V and VI were DD, II, VII and VIII were FF and pair IX was EE.

C. limon showed two distinctly heterozygous pairs (IV and VII). However, the most conspicuous chromosome markers were pair I (BB) and pair III (CC), each with two large CMA+ blocks (Fig. 2e and f). Pair IV (DD) was homozygous for band size and location but the CMA+ block of one of its homologues seemed to comprise two contiguous blocks, slightly separated, weakly positive to CMA and deeply negative to DAPI staining. Only pair VII (EF) was heterozygous for chromosome types. Pair V was DD, VI was EE and II, VIII and IX were FF.

C. limonia had the smallest number of CMA+ blocks among the species analysed here. It seemed to be heterozygous only for pair $\mathrm{I}(\mathrm{BC})$. The $\mathrm{C}$ homologue was conspicuously smaller in size (Fig. $2 \mathrm{~g}$ and $\mathrm{h}$ ). No further particular karyotype features were found. Chromosomes II, V, VII and IX were FF and III, IV, VI and VIII were DD.

In $C$. sinensis pair I (CC) was characterized by two paricentromeric bands. Its short arm showed a CMA+ /DAPI - band whereas the proximal region of the long arm was DAPI - and often stretched, such as that observed in C. aurantium (Fig. 3a and b; $\mathrm{c}$ and d). Pair III was represented by a large and a small metacentric chromosome with the same banding pattern (CC). The larger metacentric had bands proportionally larger than the smaller. Pair VIII was heteromorphic for the DF type. Pairs II, VII and IX were FF and pairs IV, V and VI were DD. Pairs IV-VIII were almost identical in size and morphology. One homologue of these showed the region near the telomeric band frequently extended like a secondary constriction during prophase (Fig. $3 \mathrm{c}$ and d).

C. medica showed a conspicuous heteromorphism on pairs I (AC) and III (BD). The C homologue of pair I had the largest CMA+ block of the complement (Fig. $3 \mathrm{e}$ and $\mathrm{f}$ ). The large terminal block of the III D chromosome was sometimes observed as two contiguous blocks, as observed in chromosome IV of $C$. limon. Pairs II, VII and IX were FF, IV, V and VI were DD and pair VIII was EE.

\section{Discussion}

According to previous measurements of satellite DNA (Ingle et al., 1973) and C-banding analyses (Guerra, 1985; Liang, 1988; Wei et al., 1988) Citrus chromosomes have a large amount of heterochromatin. The CMA/DAPI double staining revealed the occurrence of at least two different kinds of heterochromatin. The main type, a CMA+/DAPI - heterochromatin, formed terminal blocks in the long arms of most of the chromosomes. The other type, a deeply DAPIheterochromatin, was seen as either bright or faint after CMA staining. Its occurrence was restricted to only one or two chromosomes of pairs I, III and IV in each diploid set. This band was frequently found in prophase or metaphase as a stretched segment and probably represents the secondary constrictions which, as in many other species, are also deeply DAPI-negative (Schweizer, 1976; Deumling \& Greilhuber, 1982).

A third case of heterochromatin, undetectable by CMA/DAPI analysis, may also be present on many chromosomes. DAPI-stained interphase nuclei showed several bright chromocentres which had no correspondence in prophase or metaphase chromosomes. The pericentromeric regions of many chromosomes were more deeply stained than the euchromatin after $\mathrm{C}$-banding although not as darkly as the telomeric and some other proximal C-bands. In the same manner, Feulgen-stained prometaphases show many proximal blocks not observed after CMA/DAPI staining (Guerra, 1985). Wei et al. (1988) and Liang (1988) have described many proximal bands in Citrus species. Unfortunately, they do not show photographs and so their results cannot be compared. Weakly stained $\mathrm{C}$-bands have also been observed in some other plant taxa (Sato et al., 1979; Gill, 1981) and may represent a particular kind of heterochromatin.

A comparative analysis of the six karyotypes summarized in Fig. 4 shows some common characteristics in size and band distribution patterns. The first is the high degree of conservation of pair II. This pair was the only one to be homozygous and monomorphic in all species. The differences in chromosomal size between pairs I, II and III were often very small and it is possible that in some species this pair may have another position in a size-ordered karyotype (see diagrams by Liang, 1988). In addition, measurements performed on CMA pictures tend to overestimate the size of heterochromatin-rich chromosomes and hence the ordering of polymorphic pairs. It is important to keep this chromosome in the same position to conserve a homoeologous relationship among the different karyotypes (see Gill, 1981). 

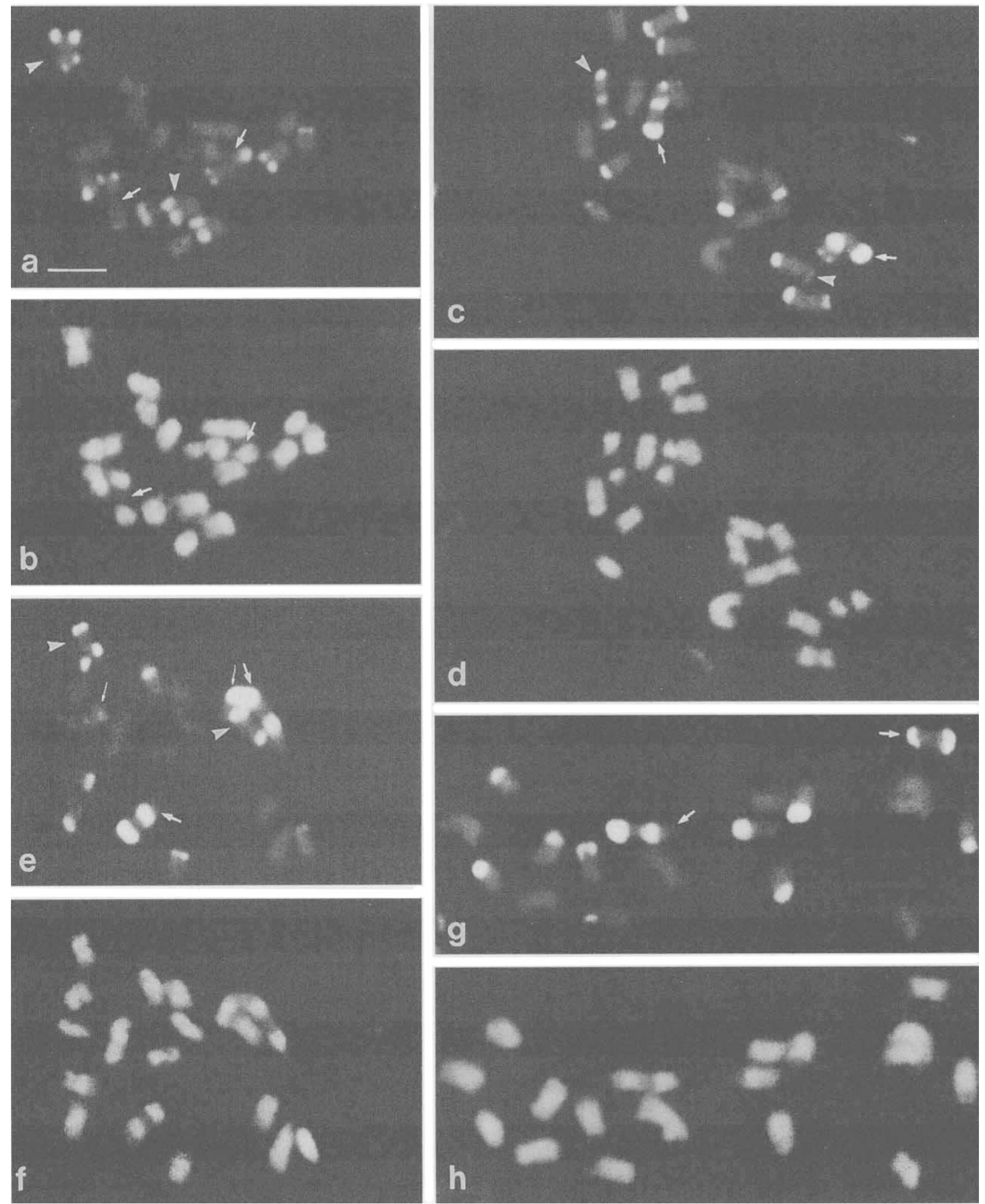

Fig. 2 CMA/DAPI-stained chromosome complements of Citrus species. (a-b) C. aurantium. (c-d) C. grandis. (e-f) C. limon. ( $\mathrm{g}-\mathrm{h})$ C. limonia. Main chromosomes are indicated by thick arrows (pair I), arrowheads (pair III) and thin arrows (pair IV). Bar represents $3.0 \mu \mathrm{m}(\mathrm{a})$. 

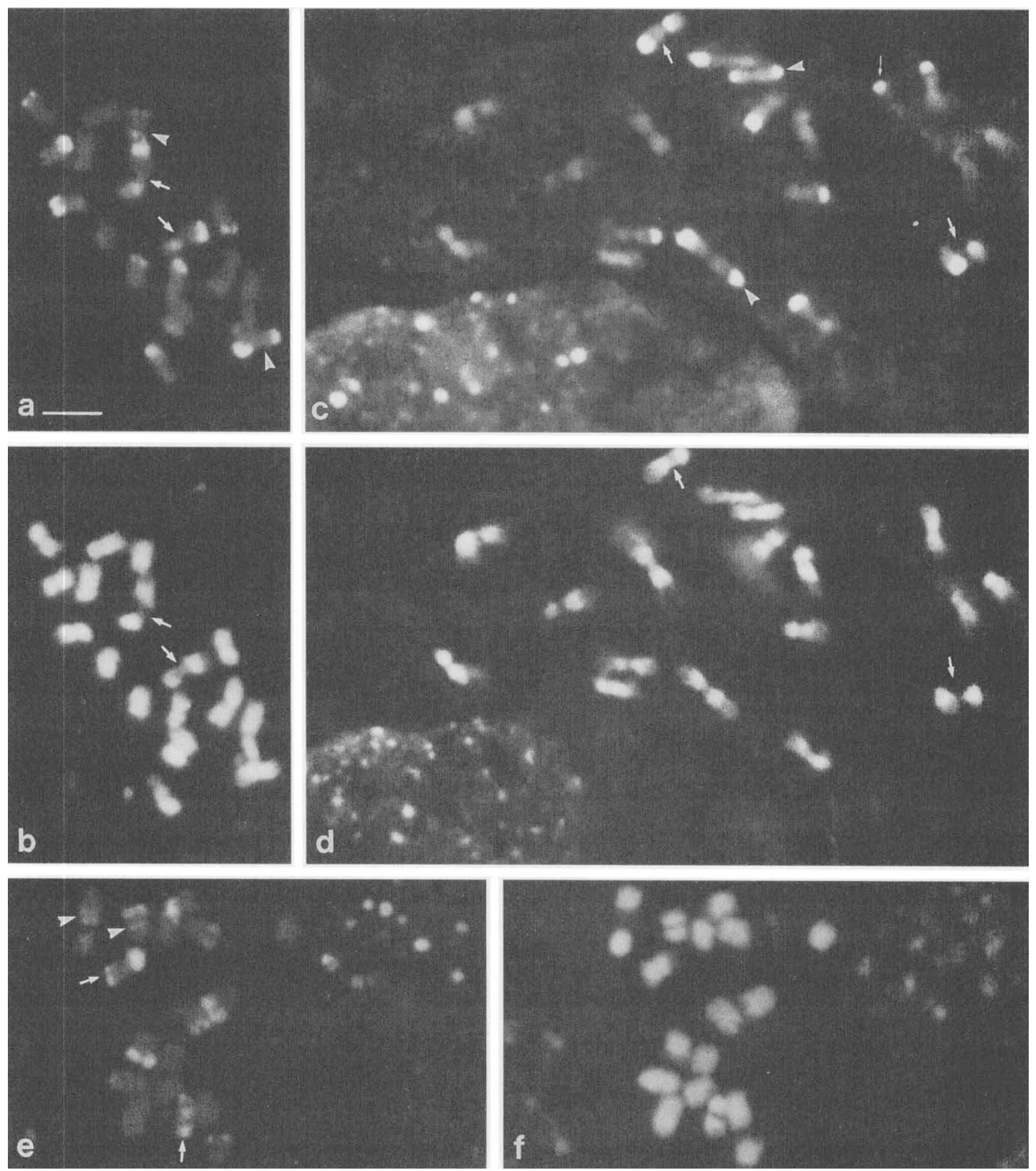

Fig. 3 CMA/DAPI-stained chromosome complements of Citrus species. (a-d) C. sinensis. (e-f) C. medica. Main chromosomes are indicated by thick arrows (pair I), arrowheads (pair III) and thin arrows (pair IV). Note CMA+ /DAPI - and CMA- / DAPI + chromocentres in the interphase nuclei (c and d; e and f). Bar represents $3.0 \mu \mathrm{m}$ (a). 
Fig. 4 Idiogram of Citrus species with their CMA/DAPI bands. Roman numbers indicate the chromosome order. The presence of two chromosomes at any point indicates heteromorphy. Interval between bars at left represents $1 \mu \mathrm{m}$. (-) CMA+/DAPIbands. ( Bright or neutral for CMA and strongly negative for DAPI. The fine telomeric band observed in some $\mathrm{E}$ chromosomes is indicated only for chromosome II because it was consistently observed in all species.

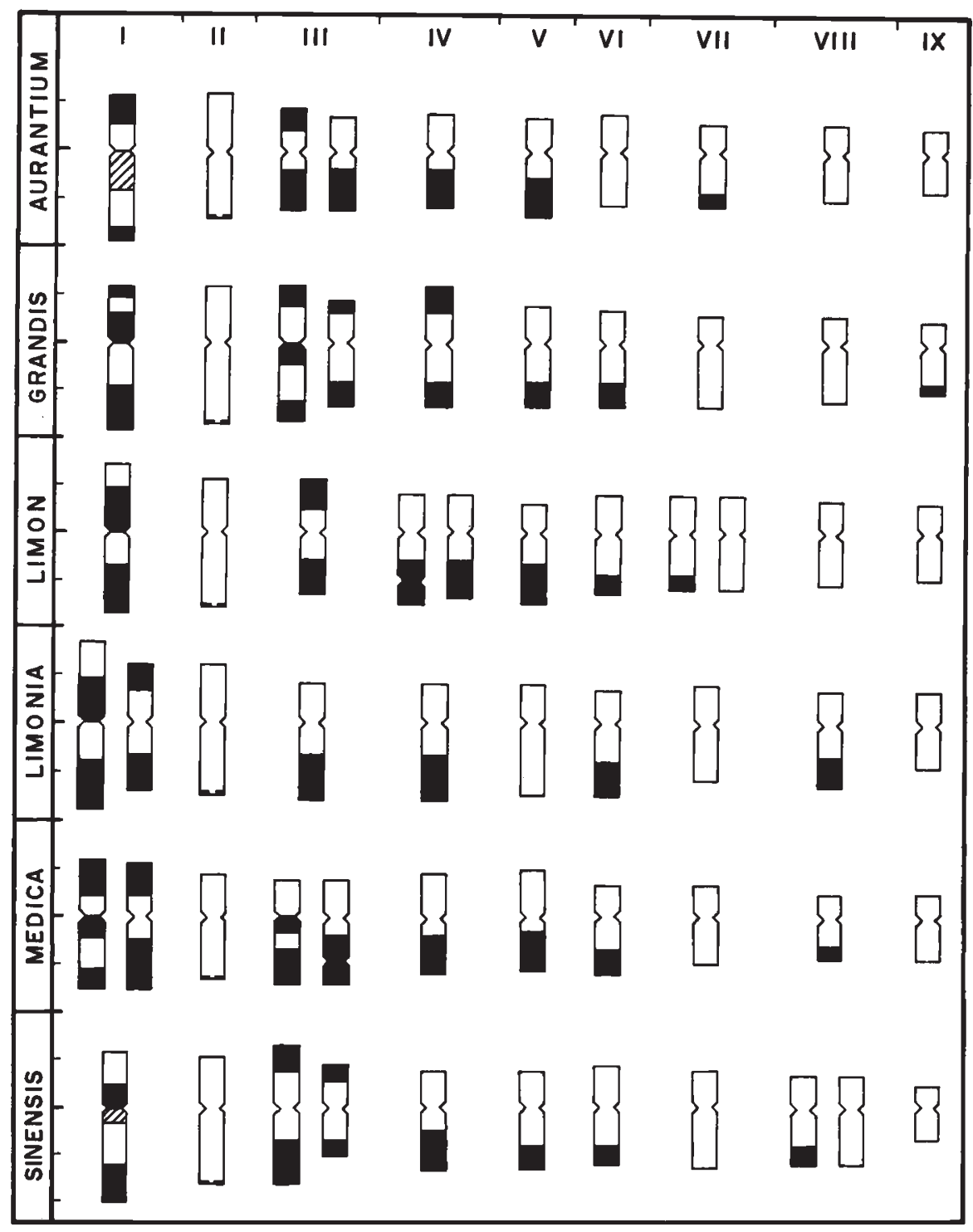

A second common feature was the higher amount of heterochromatin and high frequency of heterozygosity in pairs I and III. These were generally of the A, B, C or more seldom D types. Pair I was generally of A or B type and pair III was generally of C or D type. All other species were heterozygous for one of these pairs except for $C$. limon. The remainder were almost always homozygous.

The banding patterns of the six smaller pairs also suggest another common characteristic of the karyotype of these species. Pairs IV, V and VI were generally of D type whereas pairs VII, VIII and IX were more commonly of $\mathrm{E}$ or $\mathrm{F}$ type. This trend can be stronger still if one considers that the chromosomal banding pattern may reflect the interkaryotype homoeology better than the chromosomal size (Gill, 1981), as was considered for pair II. In this case, pairs VI and VII of
C. aurantium and V and VIII of C. limonia should have their ordering changed, enhancing the banding pattern similarity within small- and medium-sized chromosomes. Initial genetic linkage maps of citrus species showed a strong conservation in the locus order (Durham et al., 1992; Jarrel et al., 1992) that agrees largely with the observed interspecific chromosome homoeology. The skewed segregation ratios of some loci may also be explained on the basis of individual chromosomal variability and heterozygosity.

This last trend seems to support a hypothesis, based on the occurrence of meiotic secondary associations, that the basic number of Citrus should be $x=3$ (Banerji, 1954; Sharma \& Bal, 1957; Agarwal, 1987a, b). The same has been observed and claimed for some other related genera (Banerji, 1957; Agarwal, 1987b). These secondary associations, however, may result 
from the similarity in the banding pattern and chromosome size between the three medium and three small pairs. Furthermore, the radical divergence in banding pattern and evolutionary stability of pair $I$, in relation to pairs II and III, strongly suggests a lack of homoeology between the three largest pairs thus making the hypothesis of $x=3$ very improbable.

The high karyotypic diversity observed among Citrus species is quite uncommon in woody plants. Species with large karyotypic diversity, as found in Trillium (Haga, 1969), Scilla (Vosa, 1973a) and Tulbaghia (Vosa, 1973b), have exclusively or predominant sexual reproduction with high outbreeding rates and selfincompatibility. In Citrus the combination of weak interspecific crossing barriers with agamospermy seems to be the mechanism responsible for the arising and maintenance of chromosomal diversity and heterozygosity.

The CMA/DAPI banding patterns of Citrus species open up new possibilities for the use of cytological data for evolutionary analysis and breeding programmes. Preliminary observations of several Citrus-related genera have revealed that CMA/DAPI banding is a suitable approach to the cytotaxonomy of the tribe (Guerra, 1990). In relation to the chromosomal evolution of the genus, the CMA/DAPI banding pattern has brought no clear evidence of translocation or inversion as suggested by some authors (Sharma \& Bal, 1957; Raghuvanshi, 1962; Naithani \& Raghuvanshi, 1963) but it supports the putative hybrid origin of some clones and species (Moscoso \& Shambulingappa, 1972). In addition, fluorescence banding offers a powerful tool to distinguish zygotic from nucellar embryos. The identification may be done in very young seedlings and in many cases a full karyotype analysis of the offspring is not necessary. Pairs I and III, which are between the biggest and more easily identifiable, constitute reliable chromosome markers for most of the possible crosses among the species analysed here. It would also be very useful and much easier to confirm the identity of intergeneric hybrids from their higher chromosome banding divergence (Guerra, 1985, 1990; Liang, 1988).

\section{Acknowledgements}

I am very grateful to Dr Simon Bennett and Dr Peter Brandham for corrections to the English manuscript and Professor Dieter Schweizer for permission to use his laboratories at the Institute of Botany, University of Vienna. This work was supported by a CNPq grant.

\section{References}

AGARWAL, P. K. 1987a. Cytogenetical investigations in Rutaceae. I. Meiotic studies in four Citrus species of hybrid origin. Cytologia, 52, 753-756.

AGARWAL, P. K. 1987b. Cytogenetical investigations in Rutaceae. II. Meiotic studies in three intergeneric hybrids of Citrus. Cytologia, 52, 757-760.

ASHARI, S., ASPINALL, D. AND SEDGLEY, M. 1988. Discrimination of zygotic and nucellar seedlings of five polyembryonic citrus rootstocks by isozyme analysis and seedling morphology. J. Hort. Sci., 63, 495-703.

BANERJI, 1. 1954. Morphological and cytological studies on Citrus grandis Osbeck. Phytomorphology, 4, 390-396.

BANERJI, 1. 1957. A note on the cytology and pollen of Aegle marmelos Corr. Phyton, 8, 75-78.

BARRETT, H. C. AND RHODES, A. M. 1976. A numerical taxonomic study of affinity relationships in cultivated Citrus and its close relatives. System. Bot., 1, 105-136.

CAMERON, J. W. AND FRost, H. B. 1968. Genetics, breeding and nucellar embryony in citrus. In: Reuther, W., Batchelor, L. D. and Webber, H. J. (eds), The Citrus Industry, vol. II, University of California Press, California, pp. 325-366.

DEUMLING, B. AND GREILHUbER, J. 1982. Characterization of heterochromatin in different species of the Scilla siberica group (Liliaceae) by in situ hybridization of satellite DNAs and fluorochrome banding. Chromosoma, 84, 535-555.

DURHAM, R. E., LIOU, P. C., GMITTER JR., F. G. AND MOORE, G. A. 1992. Linkage of restriction fragment length polymorphisms and isozymes in Citrus. Theor. Appl. Genet., 84, 39-48.

FROST, H. B. 1925. The chromosomes of Citrus. J. Washington Acad. Sci., 15, 1-3.

HAGA, T. 1969. Structure and dynamics of natural populations of a diploid Trillium. Chromosomes Today, 2, 207-217.

GILL, B. S. 1981. Evolutionary relationships based on heterochromatin bands in six species of the Triticinae. J. Hered., 72, 391-394.

GROSSER, J. W., GMITTER, F. G. AND CHANDLER, J. L. 1988. Intergeneric somatic hybrid plants from sexually incompatible woody species: Citrus sinensis and Severinia disticha. Theor. Appl. Genet., 75, 397-401.

GUERrA, M. 1985. Cytogenetics of Rutaceae. III. Heterochromatin patterns. Caryologia, 38, 335-346.

GUERRA, M. 1987. Cytogenetics of Rutaceae. IV. Structure and systematic significance of the interphase nuclei. Cytologia, 52, 213-222.

GUERRA, M. 1990. Poliembrionia e heterozigosidade cromossômica em Citrus e gêneros afins. Resumos do XLI Congresso Nac. de Botânica. (Fortaleza): 115.

IGLESIAS, L., LIMA, H. AND SIMON, J. P. 1974. Isoenzyme identification of zygotic and nucellar seedlings in Citrus. $J$. Hered, 65, 81-84.

INGLE, J., PEARSON, G. G. AND SINCLAIR, J. 1973. Species distribution and properties of nuclear satellite DNA in higher plants. Nature New Biol., 242, 192-197.

JARREL, D. C., ROOSE, M. L., TRAUGH, S. N. AND KUPPER, R. S. 1992. A genetic map of citrus based on the segregation of isozymes and RFLPs in an intergeneric cross. Theor. Appl. Genet., 84, 49-56. 
KobaYASHI, S., OHGAWARA, T., FUJIWARA, K. AND OIYAMA, I. 1991. Analysis of cytoplasmic genomes in somatic hybrids between navel orange (Citrus sinensis Osb) and 'Murcott' tangor. Theor. Appl. Genet., 82, 6-10.

KRUG, C. A. 1943. Chromosome numbers in the subfamily Aurantioideae with special reference to the genus Citrus. Bot. Gaz., 104, 602-611.

LIANG, G. 1988. Studies on the Giemsa C-banding patterns of some Citrus and its related genera. Acta Genet. Sin., 15, 409-415.

MOORE, G. A. AND CASTLE, w. S. 1988. Morphological and isozymic analysis of open-pollinated Citrus rootstock populations. J. Hered., 79, 59-63.

moscoso, C. G. AND SHAmbulingaPPA, K. G. 1972. Cytological studies on Chironja. J. Agric. Univ. Puerto Rico, 56, 426-431.

NAITHANI, S. P. AND RAGHUVANSHI, S. S. 1963. Cytogenetical studies in Citrus. Part I. Genetica, 33, 301-312.

RAGHUVANSHI, S. S. 1962. Cytogenetical studies in genus Citrus. Iv. Evolution in genus Citrus. Cytologia, 27, 172-188.

SATO, S., KUROKI, Y. AND OHTA, S. 1979. Two types of color- differentiated C-banding positive segments in chromosomes of Nothoscordum fragrans, Liliaceae. Cytologia, 44, $715-725$.

SCHWEIZER, D. 1976. Reverse fluorescent chromosome banding with Chromomycin and DAPI. Chromosoma, 58, 307-324.

SELLITO-BOAVENTURA, Y. M. AND PIO, R. M. 1989. Análise citogenética em três cultivares de laranja doce (Citrus sinensis (L.) Osbeck). Rev. Brasil. Genet., 12, 117-126.

SHARMA, A. K. AND BAL, A. K. 1957. Chromosomes studies in Citrus. I. Agron. Lusitana, 19, 101-126.

vosA, c. 1973a. Heterochromatin recognition and analysis of chromosome variation in Scilla sibirica. Chromosoma, 43, 269-278.

vosA, C. 1973b. Quinacrine fluorescence analysis of chromosome variation in the plant Tulbaghia leucantha. Chromosomes Today, 4, 345-349.

WEI, W., CHENG, Y. AND DUAN, Y. 1988. Studies on the evolution of Citrus based on karyotype and Giemsa C-banding patterns. Acta Hort. Sin., 15, 223-228. 